\title{
PERSPECTIVA BIOÉTICA SOBRE EL ABORTO EN ARGENTINA A TRAVÉS DEL APORTE DEL CINE
}

\author{
Irene Cambra Badii ${ }^{1}$, Paula Belén Mastandrea², Delfina Martínez², María Paula Paragis²
}

Resumen: A partir de las campańas por el Aborto Legal, Seguro y Gratuito, en marzo de 2018 se debate en el Congreso argentino una propuesta sobre la Interrupción Voluntaria del Embarazo, que luego no es aprobada en el Senado. El tema adquiere una notable difusión en los medios y una participación masiva de los ciudadanos, manifestándose a favor y en contra de dicho proyecto de ley. Resulta pertinente abordar el tema del aborto en Argentina desde una perspectiva bioética que trascienda cuestiones ideológicas y morales. El presente artículo se propone analizar una película argentina contemporánea, cuyo argumento gira en torno al aborto que intenta practicar una joven de diecisiete ańos en un contexto donde es ilegal. La metodología empleada tiene un enfoque cualitativo y propone un análisis de contenido de la narrativa del film para responder dos preguntas de investigación: cómo se expone el tema del aborto en la película, desde una perspectiva bioética, y cómo se describe el contacto de la persona gestante con los profesionales de la salud. Entre los resultados encontrados se puede observar cómo la ilegalidad repercute en la decisión de la protagonista y cómo se articula con los principios bioéticos.

Palabras clave: aborto, principios bioéticos, análisis de contenido, film, profesionales de la salud

\section{Bioethical perspective on abortion in Argentina through the contribution of cinema}

\begin{abstract}
Campaigns for Legal, Safe and Free abortion have led to the discussion in March of 2018 of a proposal on voluntary termination of pregnancy at the Argentine Congress, which was not approved afterwards at the Senate. The subject acquired remarkable diffusion in the media and massive participation of citizens in the streets, standing for and against the draft legislation. In this context, it is relevant to address the issue of abortion in Argentina from the bioethical perspective aiming to transcend ideological and moral issues. This article intends to analyse a contemporary Argentine film in which the plot revolves around the attempts of a seventeen-year-old girl who tries to have an abortion in a context where it is illegal. The methodology chosen is qualitative, and proposes content analysis to answers two research questions: how is the issue of abortion exposed in the film, from a bioethical perspective, and how is the contact between the pregnant person and health professionals described. Amongst the results found, considering the basic principles of bioethical principlism, such context of illegality affects the decision of the protagonist.
\end{abstract}

Keywords: abortion, bioethical principles, content analysis, film, healthcare professionals

\section{Perspectiva bioética sobre o aborto na Argentina através das contribuiçóes do cinema}

Resumo: A partir das campanhas pelo Aborto Legal, Seguro e Gratuito, em março de 2018 se debate no Congresso argentino uma proposta sobre a Interrupçáo Voluntaria da Gravidez, que não foi aprovada no Senado. O tema adquiri uma notável difusão na mídia e uma participação maciça dos cidadãos, manifestando-se a favor e contra tal projeto de lei. Disto resulta pertinente abordar o tema do aborto na Argentina desde uma perspectiva bioética que transcenda questóes ideológicas e morais. O presente artigo se propóe a analisar uma película argentina contemporânea, cujo argumento gira em torno do aborto que uma jovem de dezessete anos tenta realizar em um contexto onde é ilegal. A metodologia empregada tem um enfoque qualitativo e propóe uma análise de conteúdo da narrativa do filme para responder duas perguntas de investigação: como se expóe o tema do aborto na película, desde uma perspectiva bioética, e como se descreve o contato da gestante com os professionais da saúde. Entre os resultados encontrados se pode observar como a ilegalidade repercute na decisão da protagonista $\mathrm{e}$ como se articula com os princípios bioéticos.

Palavras chave: aborto, princípios bioéticos, análise de conteúdo, filme, professionais da saúde

\footnotetext{
${ }^{1}$ CONICET- Facultad de Psicología, Universidad de Buenos Aires, Buenos Aires, Argentina Correspondencia: irenecambrabadii@gmail.com

${ }^{2}$ Facultad de Psicología, Instituto de Investigaciones, Universidad de Buenos Aires, Buenos Aires, Argentina
} 


\section{Introducción}

Entendiendo que el campo de la bioética se ocupa del estudio del comportamiento humano en las cuestiones relacionadas con la vida y la muerte, utilizando un abordaje multidisciplinario que contemple la dimensión social(1), resulta pertinente reflexionar sobre la práctica del aborto. Desde esta perspectiva, la misma se define como la "interrupción espontánea o intencionada del embarazo antes de que el embrión o feto se haya desarrollado(2:1)", y se lleva a cabo de distintas formas, dependiendo principalmente del marco normativo vigente en el país de la persona gestante y de los costos financieros para acceder al servicio.

Estudios recientes de la OMS demuestran que en aquellos países en donde las leyes son restrictivas respecto de la interrupción voluntaria del embarazo son mayores las tasas de abortos peligrosos, lo que implica el riesgo de complicaciones graves o incluso la muerte de la persona gestante(3). Este es el caso de Latinoamérica, donde el aborto solo está despenalizado en cuatro países - Uruguay, Guyana, Cuba y Puerto Rico-, mientras que en el resto de la región la legislación limita esta práctica de maneras diversas, que van desde la permisividad de la misma solo en casos excepcionales, hasta la prohibición absoluta.

En el mundo se producen aproximadamente 25 millones de abortos peligrosos al año, cifra que representa el $45 \%$ del total de los abortos realizados(3), lo que implica el riesgo de complicaciones graves o incluso la muerte de la persona gestante. En Argentina, el aborto inducido está tipificado como delito en el Código Penal y no existen cifras oficiales respecto de su práctica, aunque el informe CEDES(4) señala que se realizan entre 370.000 y 520.000 abortos legales y no legales por año.

A partir de la gestión de movimientos feministas, que en los últimos años han impulsado campañas por el Aborto Legal, Seguro y Gratuito en América Latina, en marzo de 2018 llega al Congreso argentino, para su debate, una propuesta sobre la Interrupción Voluntaria del Embarazo, proponiendo principalmente la legalización del aborto durante las primeras 14 semanas del proceso de gestación $(5)^{3}$. Si bien el 13 de junio el mismo se aprueba en la Cámara de Diputados, el 8 de agosto, al pasar a la Cámara de Senadores, no se aprueba y pierde la posibilidad de ser presentado hasta el año próximo.

A raíz de su tratamiento parlamentario y de su difusión masiva en el ámbito público, si bien una primera lectura de la situación parece posicionarse "a favor" o "en contra" de la práctica del aborto, desde el campo de la bioética se pueden establecer algunos aportes que excedan esa lectura de la situación y permitan generar un análisis de su complejidad. Asimismo, cada situación ha de ser vislumbrada a través de los determinantes sociales que ponen en riesgo a la salud, los que, a su vez, pueden ser categorizados como indicadores clasificados por las áreas biopsicosociales para la evaluación de existencia de riesgo a la salud de la mujer(6).

En esta línea, es posible abordar este escenario a partir de algunos de los fundamentos de la bioética principialista(7), a saber: a) autonomía, la cual corresponde a la autodeterminación o al autogobierno ejercidos por cada persona. Cada individuo, por lo tanto, tiene el derecho a decidir sobre sí; b) beneficencia, que se refiere al deber de ayudar a los otros, promover o hacer el bien, maximizando los beneficios y minimizando los riesgos; c) la no maleficencia propone la obligación de no causar daños a los pacientes o ponerlos en riesgo; y d) la justicia, que corresponde al principio formal de equidad, lo cual determina la distribución justa, equitativa y universal de deberes y beneficios sociales(8). Tal como se ha mencionado, el aborto inseguro es considerado un problema de salud pública, de derechos humanos y de justicia social, ya que su discusión se relaciona con el respeto de la dignidad de las personas gestantes, en tanto puedan hacer uso de su autonomía reproductiva en el ejercicio pleno de sus derechos(9). Tal es así que la Federación Internacional de Ginecología y Obstetricia (FIGO) promulga la defensa del derecho de la mujer a la autonomía, proponiendo el acceso a abortos seguros, sin discriminación étnica o social, entre otros puntos. Para ello, la FIGO declara que los gobernantes deben empeñarse en

\footnotetext{
${ }^{3}$ Para más información, se puede consultar el Proyecto de Ley de IVE y sus fundamentos. Disponible en: https://www.hcdn.gob.ar/ proyectos/textoCompleto.jsp? $\exp =4161-\mathrm{D}-2016 \&$ tipo $=\mathrm{LEY}$
} 
asegurar este derecho, esforzándose para desarrollar actividades educativas con el objetivo de evitar embarazos no deseados(1).

Lo que ocurre principalmente en aquellos países donde la legislación es restrictiva respecto del aborto, es que se genera un negocio en la clandestinidad, al cual solo tienen acceso quienes poseen un nivel socioeconómico elevado para atenderse en condiciones propicias y por un profesional de la salud(10). Por el contrario, las que no poseen los recursos necesarios para realizar el procedimiento en estas condiciones, suelen recurrir a métodos alternativos por fuera del modelo biomédico, los que resultan sumamente peligrosos para la integridad física y psicológica del sujeto. La falta de garantías por parte del Estado conduce a omisiones en el campo de la acción pública que redobla la sanción ya impuesta por la ley, librando a las mujeres al contexto clandestino y riesgoso. En este sentido, Donoso(11) plantea que se vuelve necesaria una integración de las distintas esferas de la vida reproductiva mediante la educación sexual, la planificación familiar y el acceso al aborto seguro, en tanto condiciones básicas de un sistema de salud equitativo y justo.

En este punto, desde la bioética, se distingue la vulnerabilidad existencial — propia de la condición humana- de la vulnerabilidad social circunstancial, específica, relacionada con la pobreza, la violencia, etc.- - A esta categorización clásica se suma, en el contexto actual, en el que se valoriza la individualidad y el pluralismo moral, la vulnerabilidad moral. Esta última abarca aquellos mecanismos de exclusión a los cuales algunos individuos y grupos se ven sometidos por divergir o transgredir valores y estándares morales hegemónicos. Se trata de situaciones sociales, pero que surgen por la fuerza de la cultura, por la visión del mundo dominante que moldea la realidad. En dicha categoría se ubican las situaciones en las cuales las personas gestantes recurren al servicio de salud para tratar consecuencias de aborto clandestino y esos servicios le son negados, o prestados de modo precario, por ser calificadas moralmente como "abortistas"; al igual que los estigmas que sufren los profesionales de la salud que las atienden en casos de abortos permitidos por ley(12).

Precisamente, en la línea de la discusión moral, en Argentina el debate suele centrarse en la condición del feto o embrión como persona $(13,14)$, como eje para determinar la permisividad o prohibición de la interrupción voluntaria del embarazo. De este modo, los argumentos que se esgrimen toman la noción de vida o de persona del campo de la filosofía y la religión, mientras que quienes asumen la posición contraria consideran cuestiones tales como los derechos sexuales y reproductivos de las personas gestantes, y la incidencia epidemiológica de muertes por la práctica de abortos clandestinos. Esta estrategia no genera un diálogo fecundo, ya que los argumentos que se proponen no pueden ser aceptados por la parte opuesta como razonables(15).

Para poder interrogar y analizar un escenario de tal complejidad desde una perspectiva bioética, se disponen de diversas fuentes que pueden servir como acceso a múltiples enfoques, entre otros, los medios de comunicación y las fuentes culturales. Dentro de estas últimas, el cine es utilizado desde su aporte narrativo y resulta una herramienta invaluable para analizar cuestiones bioéticas, considerando el valor pedagógico y científico que estos relatos culturales tienen en la sociedad en general y, particularmente, en la educación científica (16-20).

Siguiendo a Bakhtin (21), se considera que las narrativas audiovisuales constituyen un conjunto de características visuales y lingüísticas culturalmente significativas, con capacidad de revelación de una trayectoria acerca de lo que se entiende tanto por arte cinematográfico como por historia y narración. En un film no solo se halla la dimensión del relato, relacionada con la historización de la narración y su cronología, sino también la de la trama, considerada como el conjunto de teorías en las que se basa aquel que narra, sus concepciones, visiones y valores(22).

A través de un film, o de un fragmento del mismo, se puede acceder al núcleo de una problemática compleja y reflexionar sobre problemáticas ligadas con el campo de la salud, como así también considerar las incumbencias de la bioética para pensar el devenir de una historia, haciendo foco en un personaje. El material cinematográfico que se propone analizar en este artículo puede pensarse desde el paradigma de la singularidad(23), es 
decir, reflejando en una historia particular un conocimiento que puede ser compartido con otros, y constituyendo así una via regia para desplegar las cuestiones bioéticas (17). De este modo, es posible reflexionar a partir del material del film sobre el ethos situacional, entendido como los elementos morales, estéticos y valorativos de una cultura determinada(12), el cual incide sobre las prácticas y la constitución subjetiva de la protagonista.

Para tales fines, se selecciona para el análisis una producción contemporánea de Argentina: Invisible(24). La trama despliega la historia de una joven de diecisiete ańos. Al enterarse de que está embarazada de un compañero de trabajo con quien mantiene relaciones sexuales ocasionales, decide buscar la manera de llevar adelante un aborto, lo cual le resulta dificultoso por tratarse de un procedimiento ilegal en el país. El argumento del film gira en torno de los intentos que va realizando en su búsqueda, que incluyen el aborto farmacológico y una clínica clandestina.

En función de lo aquí expuesto, y a partir de la relevancia y actualidad que dicha problemática posee para el campo de la bioética, la pregunta de investigación que guía este trabajo es: ¿cómo se expone el tema del aborto en el film? Y, a su vez, ¿cómo puede leerse el contacto de la persona gestante con los profesionales de la salud a partir de los principios de bioética?

\section{Metodología}

Este trabajo, de carácter exploratorio-descriptivo, sigue las bases del enfoque cualitativo y utiliza la metodología descriptiva interpretativa $(25,26)$. Se propone un estudio de caso único para poder desplegar en profundidad una problemática bioética compleja, entendiendo que el estudio en detalle brinda la posibilidad de conocer exhaustivamente una realidad social(27). Esta estrategia de investigación hace foco en una unidad individualizada, que puede ser un grupo social, un documento o un film, para destacar sus particularidades. Teniendo en cuenta su finalidad, que es la de comprender la cuestión del aborto en tanto perspectiva bioética, el estudio de caso es de tipo instrumental(28), es decir, se trata de un instrumento para comprender esa cuestión concreta.
Se utiliza el análisis de contenido siguiendo la perspectiva de Bardin(1996), quien señala que se trata del "conjunto de técnicas de análisis de las comunicaciones tendentes a obtener indicadores (cuantitativos o no) por procedimientos sistemáticos y objetivos de descripción del contenido de los mensajes permitiendo la inferencia de conocimientos relativos a las condiciones de producción/recepción (contexto social) de estos mensajes(29:32)". Se considera la lectura e interpretación de los datos como el instrumento privilegiado para acceder al texto y al subtexto del film o documento, de modo tal de poder puntualizar las posibles condiciones de recepción de las significaciones sobre el aborto y las cuestiones bioéticas asociadas.

\section{Muestra}

Se elige al film Invisible por tratarse de la última producción argentina que aborda la temática del aborto. Su estreno fue el 8 de marzo de 2018, Día Internacional de la Mujer, en medio del debate sobre la despenalización del aborto en el país. Resulta especialmente interesante analizar dicho contexto mediante una producción actual y local, puesto que el objetivo del presente escrito es indagar los contenidos bioéticos de la exposición de determinado tema, situado en determinado tiempo y lugar. Si bien 7.000 espectadores vieron el film en las salas de cine, su audiencia ha crecido a partir de incluirse en la plataforma Netflix, y ha recibido importantes premios de prestigio internacional: mejor largometraje en el Festival de Málaga (2018) y Festival de Mar del Plata (2017), y mejor guión en el Festival de La Habana (2017) 4 .

\section{Procedimiento de recolección de los datos y va- lidez de las categorias de análisis}

Luego de determinar el objeto de análisis a través de las preguntas de investigación, se procedió a determinar las reglas de codificación, el sistema de categorías y las inferencias de lectura. El proceso de codificación ha sido llevado a cabo por cuatro investigadoras independientes, formadas en bioética y supervisadas por un quinto investigador, que trabajaron poniendo en común la delimita-

\footnotetext{
${ }^{4}$ Datos obtenidos de: https://www.otroscines.com/nota-13403-taquilla-el-cine-argentino-supero-el-millon-de-espectad y https:// www.filmaffinity.com/ar/film201169.html
} 
ción de las distintas situaciones del guión del film y la lectura del mismo en función de los materiales de bioética. Códigos similares fueron eliminados o subsumidos dentro de la misma categoría. Se llevaron a cabo dos sesiones de capacitación, de dos horas cada una, para la armonización de los criterios de codificación. Luego, el resultado del trabajo en conjunto fue discutido con un equipo de tres expertos en el área, de manera tal de supervisar los códigos propuestos y revisarlos previamente a su sistematización y lectura de los resultados.

\section{Procedimiento de análisis}

Un enfoque orientado al descubrimiento(30) y a la Teoría Fundamentada en los datos(31,32) permite desarrollar un proceso de análisis en el cual los investigadores permanecen abiertos a reinterpretaciones sucesivas de los datos recopilados para la primera pregunta de investigación, agregando los detalles de los diálogos de la trama del film o preguntas bioéticas que surgen de las escenas. Esto permite encontrar matices de situaciones en los que la complejidad bioética puede ser apreciada gracias al paradigma cualitativo, es decir, desde una comprensión epistemológica centrada en el significado y la comprensión de las acciones y sus efectos.

Tomando el análisis narrativo postulado por Fisher(33), se examina la lógica discursiva de las historias, identificando sus diversos elementos: personajes, trama, estructura narrativa y temáticas que allí se despliegan(34). Dado que el paradigma narrativo considera a la comunicación humana como histórica y situacional, a través de la observación de las coordenadas situacionales que rodean a la conducta del aborto se pone en evidencia las significaciones que éste porta, a la vez que permite distinguir elementos singulares que se sustraen de la lógica general y universalizable. Asimismo, nos interesa examinar el dispositivo estético mediante el cual la práctica del aborto se representa en la pantalla.

A fines de sistematizar la información relevada, los elementos narrativos de la primera pregunta de investigación se organizan en categorías:

Proceso de toma de la decisión del aborto: se refiere a todas aquellas acciones o diálogos que mantienen los protagonistas del film que permitan ir tras la pista de este proceso: situación del personaje principal antes de la noticia del embarazo, elementos que toma en cuenta para manifestar su voluntad de abortar, qué le señalan los personajes adyacentes, cómo es la reacción de su partenaire sexual, de qué modo se ven involucrados los profesionales de la salud y cuáles son los factores explícitos e implícitos en la toma de la decisión.

Articulación con el contexto legal: considerando el contexto legal argentino antes mencionado, se analizan cuáles son las referencias explícitas a esta cuestión, así como también alusiones implícitas en cuanto a las opciones con las que cuenta la protagonista para llevar a cabo la práctica del aborto. Además, se tendrá en cuenta los mecanismos de exclusión a los cuales se ve sometida la protagonista por el hecho de divergir o transgredir valores y estándares morales hegemónicos, lo cual se encuentra ligado al concepto de vulnerabilidad moral(12).

Asimismo, la segunda pregunta de investigación se articula en relación con categorías que responden a los cuatro principios de la bioética principialista de Beauchamp y Childress(7): autonomía, beneficencia, no maleficencia y justicia. Esto implica interpretar las interacciones de la persona gestante con los profesionales de la salud a lo largo del film, en articulación con los principios fundamentales de la disciplina.

\section{Resultados}

A partir del trabajo con las categorías establecidas, se presentan los resultados obtenidos sobre el análisis de contenido del film Invisible.

En relación con la primera pregunta de investigación, respecto de las coordenadas situacionales en torno a la práctica del aborto, en primer lugar se describe a la protagonista del film. Se trata de una joven solitaria, de diecisiete años; que va a la escuela por la mañana y por la tarde trabaja en una veterinaria ${ }^{5}$ Su madre, con quien vive, se encuentra transitando una depresión severa que le

\footnotetext{
${ }^{5}$ Respecto de esta cuestión, se recuerda que en Argentina trabajar está permitido a partir de los 16 años -mediante consentimiento de los padres o tutores (Ley n ${ }^{\circ} 26.390 / 10$ )
} 
impide salir de su casa y, por lo tanto, trabajar. La joven debe hacerse cargo de las tareas domésticas y del cuidado de su madre.

Proceso de toma de la decisión del aborto: cuando sospecha la posibilidad de estar embarazada, la protagonista acude a la guardia médica con una decisión tomada: interrumpir el embarazo. Previo a ese momento no conversa con nadie de su círculo íntimo al respecto; la decisión se toma en soledad. La médica de la guardia le aconseja que lo piense, le indica que lo hable con "el padre" o con su familia. La joven se niega, ratificando su posición. Frente a la negativa por parte de la profesional de la salud de brindar asistencia para llevar a cabo el aborto, la joven le comenta a su mejor amiga acerca de la decisión que ha tomado y juntas avanzan en la búsqueda de un método que resulte efectivo y accesible. Surge entonces la posibilidad de llevar a cabo un aborto farmacológico, para lo cual ambas adolescentes se dirigen a distintas farmacias en búsqueda de los recursos necesarios. Al conseguir la medicación necesaria, la protagonista se dirige al baño de su casa con las pastillas pero decide no tomarlas.

Su partenaire sexual es un hombre de unos cuarenta años, casado y con un hijo, que trabaja en la veterinaria con la protagonista. Cuando se entera del embarazo de la joven le dice que conoce una clínica clandestina para llevar a cabo el aborto. El día del turno para la intervención, la lleva hasta la puerta del lugar y no la acompaña dentro. La joven ingresa a una de las salas pero cuando la dejan a solas decide irse. Espera un tiempo en la recepción del lugar y luego vuelve al auto con su partenaire, sin mencionar la nueva decisión que ha tomado, haciéndole creer que el aborto se ha realizado.

Articulación con el contexto legal: observamos una marcada ilegalidad respecto de la práctica del aborto en la guardia del hospital, la segunda farmacia en la que consulta sobre el fármaco para la interrupción del embarazo y, finalmente, la clínica clandestina donde se llevaría a cabo la práctica. En el hospital se explica a la protagonista que en la Argentina la interrupción voluntaria del embarazo es ilegal y que por lo tanto no pueden ayudarla a llevarla a cabo. En la farmacia se le explica que el fármaco no puede venderse sin receta. Más tarde, uno de los empleados farmacéuticos le ofrece el medicamento en forma ilegal, entregando el producto fuera del negocio, de noche, a cambio de dinero en efectivo. La encargada de la clínica clandestina se reúne con la protagonista en un bar y le describe: "la intervención la hacemos nosotros, es muy profesional. Tenés los mismos cuidados que en una clínica", luego se explica que el personal está compuesto por ella, un anestesiólogo y un doctor ginecólogo, "con título y todo". Se establece el pago correspondiente a la intervención, el cual debe ser en el momento y en efectivo.

Por otra parte, en un análisis transversal a los elementos ya mencionados, es posible ubicar en las interacciones de la persona gestante con los profesionales de la salud a lo largo del film algunas cuestiones referidas a los principios fundamentales de la bioética(7). Para efectos de sistematizar dichos elementos, se propone el siguiente ordenamiento:

Principio de autonomía: la decisión de abortar se toma en soledad, sin coerción de ningún otro sujeto. Respecto de la práctica del aborto, el contexto de ilegalidad hace que el mismo deba ser pensado en la ilegalidad, abonando una gran cantidad de dinero para ello. Tras no haber consumido las pastillas y comentarle la situación a su partenaire sexual, el mismo se ofrece a cubrir los gastos del procedimiento y le facilita un dato para que se comunique con la clínica clandestina. Cuando la protagonista decide no proseguir con la intervención, la encargada le dice que no puede devolverle el dinero. Si bien dichos intercambios se producen en el contexto de la clandestinidad, la protagonista finalmente determina por sí misma que no realizará el procedimiento quirúrgico.

Principio de beneficencia: en un primer contacto en una guardia médica, una profesional de la salud aparece de espaldas a la cámara haciéndole preguntas técnicas sobre su estado: “¿Cuándo fue tu última menstruación? ¿Cuánto llevas de atraso? ¿Te hacés atender por algún ginecólogo regularmente?". Le indica realizar una ecografía, un análisis de sangre que descarte la presencia de HIV y confirme el embarazo, y que pida turno con un obstetra para hacer seguimiento por consultorio. El foco radica, entonces, en la prevención y el cuidado de la salud física de la paciente. No se brinda psico- 
educación en esta instancia, ni tampoco se indaga sobre sus circunstancias educativas y laborales, su red de contención familiar, entre otras cuestiones.

Principio de no maleficencia: la profesional de la salud que atiende a la protagonista en la guardia le indica que realice los controles obstétricos de rutina y consulte con el equipo de salud mental. Un segundo contacto con un profesional de la salud ocurre a partir de la búsqueda en internet que realiza amiga de la protagonista, donde encuentra el dato sobre el aborto farmacológico. En un primer intento de las jóvenes, el farmacéutico no acepta venderles el medicamento sin prescripción médica. Luego acuden a otra farmacia en la que se produce un tercer contacto con un profesional de la salud, que acepta vender el medicamento sin receta, pero de forma ilegal, pautando un encuentro por fuera de la farmacia. Principio de justicia: la protagonista acude a un hospital público para realizar los estudios de sangre que constatan el embarazo. Al finalizar la consulta, afirma: "no lo voy a tener", frente a lo cual la profesional reacciona preguntándole por la opinión del padre y si ha puesto a su familia en conocimiento de la situación. Cuando la protagonista ratifica su decisión, la profesional de la salud brinda información sobre los recursos con los que cuenta, dado que la interrupción voluntaria del embarazo no puede llevarse a cabo en su caso de forma legal: "el aborto está penado por ley, salvo algunos casos muy especificos, que no sería el tuyo (...) A esta altura, si no lo querés tener, la única opción es darlo en adopción legal'. Luego la deriva al grupo de psicólogos; sin embargo, no tenemos noticias de que la consulta se lleve a cabo.

\section{Discusión}

El presente estudio analiza el contenido bioético del film argentino Invisible, que introduce la historia de una joven que intenta realizar un aborto en un contexto donde esta práctica es ilegal. Se ha recurrido a la narrativa cinematográfica, puesto que se configura como un relato cargado de valor simbólico, en el cual se representan y construyen las identidades sociales y personales de una época determinada. Si bien el análisis de contenido comúnmente soslaya el contexto en el cual la producción narrativa tiene lugar, se propone considerar el cine como "máquina cultural que traduce activamente la historia y la dinámica social(35:111)". Su operatoria consiste precisamente en señalar, mostrar, llamar nuestra atención sobre cuestiones de la vida humana(36), lo cual permite estudiar en detalle y con mayor profundidad algunos aspectos del tema seleccionado.

El diseño metodológico facilita la socialización de los datos recabados, teniendo en cuenta que nuestro objetivo radica en generar una propuesta de trabajo con profesionales de la salud —médicos y psicólogos, específicamente-que funcione a modo de capacitación en el campo de la educación en bioética. Apuntamos a que los filmes funcionen como disparadores hacia la discusión, guiando la reflexión sin brindar respuestas acabadas sobre la problemática.

Para el análisis del film se propusieron dos preguntas de investigación. El desarrollo de la primera permitió vislumbrar que el marco de ilegalidad en el cual se inscribe la práctica del aborto en la Argentina influye sobre el proceso de toma de decisión de la protagonista, limitando sus opciones en relación a la interrupción o prosecución del embarazo. Por ello, partiendo de dichos resultados, destacamos una serie de cuestiones para la ponderación de la situación.

Entre las consideraciones bioéticas que ofrece Invisible en relación con el aborto, es preciso situar, en primer lugar, que el film presenta elementos morales, estéticos y valorativos de la cultura argentina en la actualidad. El periplo que la protagonista inicia, en busca de algún procedimiento que le permita poner fin a su embarazo, pareciera estar impulsado por los mecanismos de exclusión a los que es sometida, al querer transgredir valores y estándares morales hegemónicos(12) que le impiden el acceso a la práctica en un hospital, de forma legal y segura. Por otra parte, es necesario situar que en Argentina la religión oficial es la Católica Apostólica Romana, y que, si bien existe la libertad de culto, se trata de una influencia insoslayable en la determinación de los valores sociales, por ejemplo, en relación con el aborto.

En el caso de la protagonista del film, podemos conjeturar que la forma protocolar y burocrática de proceder de la profesional de la salud de guardia resulta un indicador de la incapacidad del 
sistema sociosanitario para dar respuesta a acontecimientos que exceden el campo de la moral imperante. A su vez, es necesario hacer énfasis en la ausencia del Estado en el film, en cuanto al resguardo del bienestar de los ciudadanos(15). La protagonista se encuentra sola frente a su situación y no obtiene ayuda de ningún profesional responsable, sino que solo la aconsejan su mejor amiga, que tiene su misma edad, y su partenaire sexual. Resulta evidente que las diversas instituciones y efectores de la salud a los que la joven recurre tienen respuestas expulsivas frente a su solicitud, o bien buscan un rédito económico, dado el mercado clandestino generado en torno a la ilegalidad de la interrupción del embarazo en este país. En este sentido, se expresa la vulnerabilidad moral(12) que atañe a la protagonista por el simple hecho de querer interrumpir su embarazo.

Asimismo, observamos que la vulnerabilidad social se manifiesta a lo largo de todo el film, en relación a la falta de contención familiar y estabilidad económica de la protagonista. Una de las variables que complejiza la situación es la imposibilidad de la adolescente de continuar con el vínculo laboral a causa del embarazo, ya que, si bien no es explícito el motivo, se observa que renuncia a su empleo luego de decidir no realizar la intervención - lo cual puede interpretarse también como un intento de evitar que su partenaire sexual descubra esta situación-. Las coordenadas educativas siguen la misma línea: recordemos que se encuentra cursando su último año de secundaria y el embarazo y posterior crianza del niño sin ayuda podría poner en peligro la finalización de sus estudios.

Por otro lado, si se llevase a cabo la práctica del aborto en el film, la misma se daría en condiciones de riesgo(11), ya que, por tratarse de un procedimiento penalizado en este país, la protagonista solo puede recurrir a procedimientos practicados por personas no calificadas, en un ambiente sin las condiciones mínimas de seguridad sanitaria e higiene, o bien hacerlo ella misma. De hecho, su partenaire sexual la insta claramente a abortar en una clínica clandestina, ofreciéndole el dinero necesario a tales fines, siendo funcional al negocio que se genera en torno a esta práctica en la clandestinidad(10).
Estas coordenadas de la película pasan a ser parte de un tratamiento complejo, social, dinámico - y no menos singular- de la temática del aborto en Argentina. Se refleja el hecho de que el aborto inseguro constituye un problema de salud pública, fundamentalmente debido a la gran cantidad de muertes que conlleva su práctica, además de poner en evidencia cuestiones relativas a los derechos humanos y la justicia social, ya que lo que está en discusión se relaciona con el respeto de la dignidad de las personas gestantes, en tanto puedan hacer uso de su autonomía reproductiva en el ejercicio pleno de sus derechos(9). ¿Qué consideraciones es preciso tener presentes para la reflexión en el campo de la bioética?

El contexto legal de la Argentina respecto de la práctica del aborto posee una incidencia fundamental en la posibilidad de decidir de forma autónoma por parte de la protagonista. Los resultados permiten vislumbrar que operan circunstancias de ilegalidad en tres contactos con profesionales de la salud: la guardia del hospital, la segunda farmacia en la que consulta y la clínica clandestina. En principio, la intervención de la primera médica no se enmarca en el protocolo argentino vigente para la atención integral de las personas con derecho a la interrupción legal del embarazo, de 2015, que incluye el fallo F.A.L.(37). Allí se especifica que toda persona gestante puede solicitar esta práctica sin necesidad de autorización judicial cuando el embarazo representa un peligro para la vida o la salud de la mujer, o cuando el embarazo proviene de una violación. En este sentido, la profesional de la salud no opera en la vía de indagar las circunstancias en las cuales la adolescente ha quedado embarazada, o el estado de salud en el cual la misma se encuentra - considerando el concepto de "salud" según la definición de la $\mathrm{OMS}^{6}(38)$ _ y asume que el suyo no es un caso comprendido dentro de la ley. Asimismo, esta intervención no promueve realmente la realización de una consultoría efectiva que acompañe a la adolescente en el proceso de toma de su decisión. Por otro lado, el farmacéutico también opera en la ilegalidad, al venderle un medicamento sin prescripción médica, enfatizando el carácter clandestino del hecho al citar a la joven por fuera

${ }^{6}$ En la Declaración de Alma Ata (1978), la Organización Mundial de la Salud define a la salud como un estado de completo bienestar físico, mental y social, y no solo como la ausencia de enfermedad. 
de la farmacia para la entrega del medicamento. Finalmente, como su denominación lo indica, la clínica es "clandestina", es decir, opera por fuera de la legislación vigente en Argentina y de la regulación del Ministerio de Salud para la habilitación de los espacios que brindan estos servicios.

Por otra parte, resulta interesante volver a mencionar el dato de que la protagonista tiene diecisiete años, y que se trata de una joven que está finalizando la escuela secundaria. Según la Convención Internacional sobre los Derechos del Niño, a la cual Argentina suscribe, los nińos y adolescentes son considerados en su especificidad y desarrollo gradual como ciudadanos, por lo que se contempla el desarrollo efectivo y progresivo de la autonomía personal, social y jurídica. El paradigma actual supone que se piense al adolescente como sujeto de derechos, con inquietudes y problemáticas propias, y se establezca un límite a la "tiranía de la familia" y la "tiranía del Estado"(39). En este punto, se evidencia que los profesionales de salud que vemos en el film ignoran dichos preceptos, especialmente la efectora de salud de la guardia hospitalaria, quien rápidamente desdeña la problemática que la joven presenta e inquiere sobre la opinión de la familia.

Todos los elementos hasta aquí mencionados evidencian la complejidad que la cuestión del aborto reviste, lo cual se expone en el film. En primer lugar, una variable constante que subyace a la trama es el posicionamiento dicotómico en relación con estar a favor o en contra de esta práctica, que no solo responde a consideraciones ideológicas y religiosas, sino que se encuentra determinado también por la dificultad que representa para la sociedad hablar de esta temática. Precisamente, Invisible se propone abordar esta problemática, pero finalmente el aborto no se realiza en el film, lo cual dista de ser casual. Más allá de la dificultad que podemos inferir, respecto de exponer en escena un procedimiento como éste, el film presenta una serie de situaciones en las que se observa claramente el riesgo que comporta para la protagonista no contar con un marco seguro y legal que le permita acceder a una práctica que resguarde su salud, lo cual la deja expuesta a posibles complicaciones, al llevar a cabo prácticas sin supervisión ni cuidado profesional.
Por otra parte, respecto del análisis de la segunda pregunta de investigación, se ha hecho especial foco en los cuatro principios de la bioética, a fines de precisar cómo se llevan a cabo las consultas con los profesionales de la salud en el marco de la práctica del aborto. Teniendo en cuenta lo desarrollado en relación a la autonomía, cabe señalar aquí los otros tres principios implicados en la atención de los profesionales de la salud. En primer lugar, se retoma el principio bioético de justicia, que corresponde al principio formal de equidad, lo cual determina la distribución justa, equitativa y universal de deberes y beneficios sociales(8). En este sentido, el film evidencia la inequidad con respecto a la práctica del aborto en la clandestinidad, la cual puede darse de diversos modos más o menos riesgosos para la salud de la gestante. En la consulta con la profesional de la salud de la guardia no se le da el trato pertinente, en tanto se ofrece información escasa y no se brinda contención psicoafectiva. Asimismo, el film hace explícito que la protagonista necesita de grandes sumas de dinero para poder acceder a los dos intentos ilegales, tanto farmacológico como en la clínica clandestina. Los determinantes sociales de la salud explican la mayor parte de las inequidades sanitarias, esto es, de las diferencias injustas y evitables observadas en y entre los países en lo que respecta a la situación sanitaria(40).

En relación con los principios de beneficencia y no maleficencia, pueden considerarse en forma complementaria, ya que las indicaciones de los profesionales de la salud no contemplan los intereses de la paciente. En el primer caso, se hace evidente que la médica deja entrever ciertos prejuicios de su parte, eliminando de plano la posibilidad de abortar. Por otra parte, ninguno de los profesionales de la salud implicados parece tener en consideración los efectos a nivel psicológico que la situación acarrea para la protagonista. Sin embargo, podríamos ubicar en la intervención del primer farmacéutico una primacía del precepto primum non nocere, es decir, no hacer daño al paciente, en este caso, resguardando la autoadministración del fármaco sin seguimiento médico.

El presente trabajo de investigación pone de relieve las tensiones suscitadas en el campo de la bioética y la práctica profesional en materia de la interrupción voluntaria del embarazo, preten- 
diendo abrir interrogantes al respecto y no brindar respuestas que pudieran obturar el ejercicio de reflexión. Sin embargo, por tratarse de una temática en extremo compleja y cuyo tratamiento se enmarca en un contexto de controversia a nivel social y legislativo, futuras investigaciones podrían retomar esta problemática con las demás producciones argentinas que aborden la cuestión del aborto, o bien producciones internacionales en las que el contexto implique que el aborto sea una práctica legal, de forma tal de poder establecer una comparación desde el punto de vista de la teoría del Derecho y las implicaciones bioéticas en la relación médico-paciente y en el devenir de la persona gestante.

Asimismo, las implicaciones teóricas y prácticas de analizar cuestiones bioéticas en escenarios específicos de la práctica pueden determinarse en un futuro estudio empírico en el que se examine la eficacia pedagógica, ya que nuestros resultados demuestran que el film Invisible contiene un alto contenido de cuestiones bioéticas interesantes para la capacitación y formación de los profesionales de la salud.

\section{Agradecimientos}

Este trabajo fue realizado en el marco de los siguientes proyectos de investigación: UBACyT 20020170200229BA "Pensar el cine. La narrativa de películas y series como matriz metodológica para el tratamiento de problemas complejos" (2018-2019; Directora: Dra. Irene Cambra Badii) y UBACyT 20020170100758BA “(Bio)ética y Derechos Humanos: la dialéctica de lo particular y lo universal-singular a través de la narrativa clínico-cinematográfica” (2018-2020; Director: Dr. Juan Jorge Michel Fariña). 


\section{Referencias}

1. De Souza J, Vitório de Silva V, Santana Barbosa da V, Lozado, YA, Eliane dos Santos B, Alves J, Pereira B, Nagib E, Narriman Silva de Oliveira R. Dilemas bioéticos en la asistencia médica a adolescentes embarazadas. Revista Bioética [Internet]. 2018 [Consultado 25 de Sep 2018]; 26(1): 87-94. Disponible en: https://dx.doi.org/10.1590/198380422018261229

2. Torres Acosta R. Glosario de Bioética. 1ª ed. La Habana: Ciencias Médicas; 2011.

3. Organización Mundial de la Salud (OMS). Comunicado de prensa conjunto de la OMS y el Instituto Guttmacher. [Internet]. Ginebra: 28 de Sep de 2017 [Consultado 25 de Sep 2018]. Disponible en: http://www.who.int/es/news-room/ detail/28-09-2017-worldwide-an-estimated-25-million-unsafe-abortions-occur-each-year (2017).

4. CEDES. Situación del aborto en Argentina. [Internet]. 2005. [Consultado 25 de Sep 2018]. Disponible en: https:// www.defensorba.org.ar/micrositios/ovg/pdfs/Situacion-del-Aborto-en-Argentina.pdf

5. Proyecto de Ley no promulgado: Interrupción Voluntaria del Embarazo y sus fundamentos. Congreso de la Nación Argentina (2018). Disponible en: https://www.hcdn.gob.ar/proyectos/textoCompleto.jsp?exp=4161-D-2016\&tipo=LEY

6. Bassini C, Cruz M, Carulli C, Mahffuz R, y Dosso D. Instrumento para la evaluación de riesgo para la mujer a causa del embarazo. En: Libros de ensayos. FUSA AC. Buenos Aires, Argentina; 2017: 31-34.

7. Beauchamp TL, Childress JF. Principios de Ética Biomédica. Barcelona: Masson; 1979.

8. UNESCO. Declaración universal sobre Bioética y Derechos Humanos. [Internet]. 19 de Octubre de 2005 [Consultado 25 de Sep 2018]. Disponible en: http://portal.unesco.org/es/ev.php-URL_ID=31058\&URL_DO=DO_TOPIC\&URL_ SECTION=201.html

9. Gómez Dávila JG. El aborto: una mirada desde la salud pública, los derechos y la justicia social. Revista Colombiana de Obstetricia y Ginecología [Internet] 2018 [Consultado 25 de Sep 2018]; 69(1): 53-64. Disponible en: http://dx.doi. org/10.18597/rcog.3009

10. Tajer D. La clandestinidad del aborto y la salud mental de las mujeres. Revista ISALUD 2018; 13(61): 14-16.

11. Donoso Sabando C. Despenalización del aborto en Chile. Una cuestión de justicia social. Acta Bioethica [Internet] 2016 [Consultado 25 de Sep 2018]; 22(2): 159-167. Disponible en: https://actabioethica.uchile.cl/index.php/AB/ article/view/43753/45790

12. Sanches MA, Mannes M, Cunha da TR. Vulnerabilidade moral: leitura das exclusôes no contexto da bioética. Revista Bioética [Internet] 2018 [Consultado 25 de Sep 2018]; 26(1): 39-46. Disponible en: https://dx.doi.org/10.1590/198380422018261224

13. Herrera M, Salituri Amezcua MM. El derecho de las familias desde y en perspectiva de género. Revista de Derecho 2018; (49): 43-76.

14. Bladilo A, de la Torre N, Herrera M. Las técnicas de reproducción humana asistida desde los derechos humanos como perspectiva obligada de análisis. Revista IUS [Internet] 2017 [Consultado 25 de Sep 2018]; 11(39): 1-29. Disponible en: http://www.scielo.org.mx/scielo.php?script=sci_arttext\&pid=S1870-21472017000100002\&lng=es\&tlng=es.

15. Figueroa García-Huidobro R. La moralidad del aborto: ampliando la discusión. Acta Bioethica 2013; 19(1): $105-112$.

16. Mainetti JA. Bioética Narrativa. En: Tealdi JC, editor. Diccionario latinoamericano de bioética. Bogotá: UNESCO - Red Latinoamericana y del Caribe de Bioética: Universidad Nacional de Colombia; 2008: 167-168.

17. Michel Fariña JJ, Solbakk JH. (Bio)ética y cine. Tragedia griega y acontecimiento del cuerpo. 1a ed. Buenos Aires: Letra Viva; 2012.

18. Moratalla TD. Bioética y Cine. De la narración a la deliberación. $1^{\text {a }}$ ed. Madrid: San Pablo; 2011.

19. Moratalla TD, Feito Grande L. Bioética narrativa. $1^{\text {a }}$ ed. Madrid: Escolar y Mayo; 2014.

20. Gracia D, Muñoz S. Ética narrativa y hermenéutica. En: Médicos en el cine. Dilemas bioéticos: sentimientos, razones y deberes. Madrid: Complutense; 2006.

21. Bakthin MM. A cultura popular na idade média e no renascimento: o contexto de François Rabelais. $7^{\text {a }}$ ed. São Paulo: Hucitec; 2010.

22. Todorov T. Crítica de la crítica. 1a edición. Barcelona: Paidós; 2006.

23. Agamben G. Signatura rerum. Barcelona: Anagrama; 2010.

24. Miller JP, Rotter A, Giorgelli P. Invisible [cinta cinematográfica]. Argentina: Tarea Fina, Good Fortune Films, AireCine e Instituto Nacional de Cine y Artes Audiovisuales (INCAA) (support); 2017.

25. Sandelowski M, Barroso J. Classifying the findings in qualitative studies. Qualitative health research 2003; 13(7), 905923.

26. Thorne S. Interpretive description: qualitative research for applied practice. New York: Routledge; 2016. 
Perspectiva bioética sobre el aborto en Argentina a través del aporte del cine - Irene Cambra Badii et al.

27. Yin R. Case Study Research. Design and Methods. 4a ed. New York: Sage; 1995.

28. Stake RE, Denzin NK, Lincoln YS. Strategies of qualitative inquiry. Case studies. New York: Sage; 1998.

29. Bardin L. Análisis de contenido. Buenos Aires: Akal; 1996.

30. Creswell P. Qualitative inquiry and research design: choosing among five approaches. Thousand Oaks: SAGE Publications; 2018.

31. Glaser BG, Strauss A. The discovery of grounded theory: Strategies for qualitative research. Chicago: Aldine; 1967.

32. Strauss A, Corbin J. Grounded theory methodology. Handbook of qualitative research 1994; 17: 273-85.

33. Fisher WR. The narrative paradigm: An elaboration. Communication Monographs 1985; 52: 347-367.

34. Cullum-Swan BETS, Manning P. Narrative, content, and semiotic analysis. In Handbook of qualitative research. New York: Sage; 1994: 463-477.

35. Gómez Ponce A. Pequeńas grandes mentiras. Narraciones seriales en torno al American Dream. Representaciones [Internet] 2017 [Consultado 25 de Sep 2018]; 13(2): 107-126. Disponible en: https://revistas.unc.edu.ar/index.php/repr/ article/view/19560/19348

36. Será María J, Goitia FC. Reflexiones sobre el cine. San Fernando: Real Academia de Bellas Artes; 1990

37. Fallo de la Corte Suprema de Justicia de la Nación: "F. A. L. sobre medida autosatisfactiva”. 13 de marzo de 2012 Disponible en: http://sjconsulta.csjn.gov.ar

38. Organización Mundial de la Salud (OMS). Declaración de Alma Ata. Kazajistán; 1978.

39. Herrera M. Ensayo para pensar una relación compleja: sobre el régimen jurídico de la capacidad civil y representación legal de niños, niñas y adolescentes desde el principio de autonomía progresiva en el Derecho argentino, en Justicia y Derechos del Niño. Santiago de Chile: Fondo de las Naciones Unidas para la Infancia. 2009; (11).

40. Organización Mundial de la Salud. OMS. Informe de la Comisión sobre Determinantes Sociales de la Salud [Internet]; 2008 [Consultado el 08 de Ago de 2018]. Disponible en: http://www.who.int/social_determinants/es/

Recibido: 28 de septiembre de 2018

Aceptado: 17 de octubre de 2018 